\title{
The Metascalar Nature of Information
}

\author{
Ron Cottam $^{1}{ }^{*}$, Willy Ranson ${ }^{2}$ and Roger Vounckx ${ }^{1}$ \\ ${ }^{1}$ The Living Systems Project, Dept. of Electronics and Informatics, Vrije Universiteit Brussel (VUB), \\ Pleinlaan 2, 1050 Brussels, Belgium \\ ${ }^{2}$ IMECvzw, Kapeldreef 75, 3001 Leuven, Belgium
}

E-Mails: ricottam@etro.vub.ac.be; wranson@etro.vub.ac.be; rvounckx@etro.vub.ac.be

* Author to whom correspondence should be addressed; Tel.: +32-2-629-2933; Fax: +32-2-629-2883

Accepted:

\begin{abstract}
Brenner [1] has published a logical characterization of Nature in which ' $A$ ' and 'non- $A$ ' are nonseparable, where ' $A$ ' and 'non- $A$ ' are not the terms of standard bivalent logics but refer to elements of evolving processes or systems, and in which a third ' $\mathrm{T}$-state' can or will emerge from their interaction in terms of actual and potential. This leads also to the non-separability of ontology and epistemology. We accept that Brenner's characterization is valid, and have previously addressed [2] its relationship to the representation of hierarchical structure, following the insistence of Havel [3] that scale should be presumed to be a necessary constituent of all theory. In this paper we extend our treatment to the domain of information, which we picture as a coupling between structure and process. Hierarchy is naturally partially birational, between entity and context, and we find that this duality matches Brenner's characterization.

As indicated in Figure 1, the directly-inaccessible duality of scaled data and its scaled context presents itself as the precursor of a partial hyperscalar duality which is 'the real contextual nature' [4] of the entity in its temporal and spatial context. These two partial hyperscales self-re-integrate resulting in a singular metascalar T-state which is information. Representation is then the abductive metascalar interpretation of the unification of the dual partial hyperscales which constitutes information.

Metascalar information is both objective and subjective, in indivision. The first, entity partial hierarchy is of individual subjective scales of data, while the second is of individual subjective scales of context; a scaled form of von Uexküll's [5] umwelts. Havel [3] has presented the notion that scientific 'objectivity' is in fact societal group subjectivity. The two partial hyperscales are 'objective'
\end{abstract}


in Havel's group-subjective sense. We hypothesize that this duality is at the roots of the real and imaginary parts of evolved complex algebra, in a manner similar to the way that inter-scale data-loss is at the roots of the evolved hierarchical nature of mathematical equations.

Figure 1. Scale, context, hyperscale and metascale in a hierarchical system.

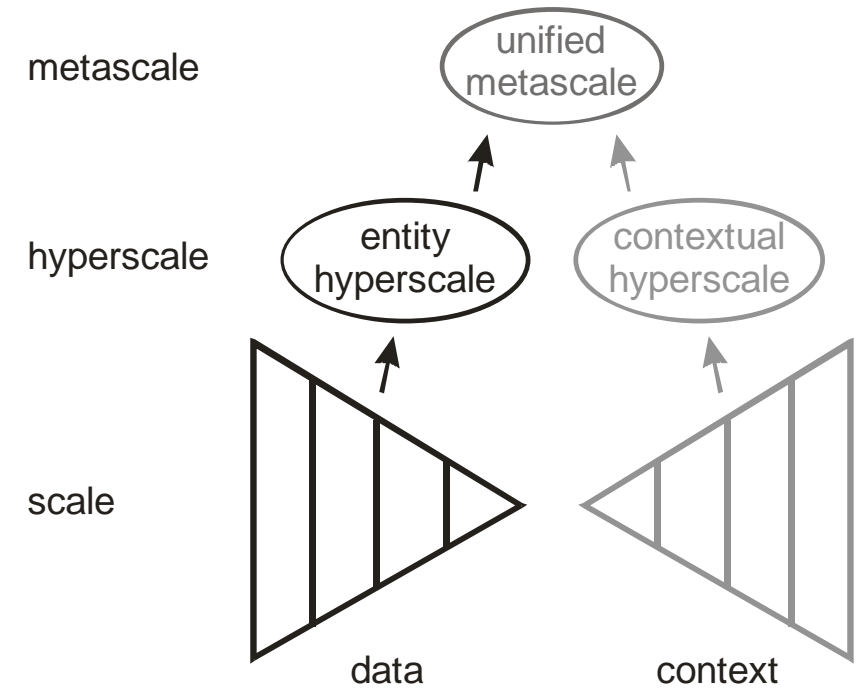

This assembly of ideas is at this level of description purely abstract: if we now look at an embodied form we find that intelligence is the tool which enables inter-scalar transitions; that sapience permits conclusions from complete scale-assemblies (the construction of hyperscale); and that embodied metascalar information is metamorphosed into the high-level informational construct of wisdom. We have described in [6] how inter-viewing between the two hyperscales generates the unified high level awareness of consciousness. This phenomenon is concurrent with the development of wisdom: the one (wisdom) is a simplistic integration of the characters and contents of the two hyperscales [2]; the other (consciousness) is a less direct integration [6] whose character depends on the interpretation of observation as a mutual measurement [7] and whose content is restricted to rapidly-changing effects by stasis neglect [6] and habituation [8]. The one is not possible without the other, and their intertwining depends on the metascalar integration of information.

\section{References}

1. Brenner, J.E. Logic in Reality; Berlin: Springer: Berlin, Germany, 2008.

2. Cottam R.; Ranson W.; Vounckx R. A framework for computing like Nature. In Computing Nature; Dodig-Crnkovic, G., Giovagnoli, R., Eds.; Springer SAPERE series: Berlin, Germany, 2013; pp. 23-60.

3. Havel, I.M. Scale dimensions in nature. International Journal of General Systems 1995, 23, 303332.

4. Cottam, R.; Ranson, W.; Vounckx, R. Autocreative hierarchy II: dynamics - self-organization, emergence and level-changing. In International Conference on Integration of Knowledge Intensive Multi-Agent Systems; Hexmoor, H., Ed.; IEEE: Piscataway, NJ, USA, 2003, pp. 766773. 
5. von Uexküll, T. Introduction: The sign theory of Jakob von Uexküll. Semiotica 1992, 89, 279315.

6. Cottam, R. and Ranson, W. A biosemiotic view on consciousness derived from system hierarchy. In The Unity of Mind, Brain and World: Current Perspectives on a Science of Consciousness; Pereira Jr., A.; Lehmann, D., Eds.; Cambridge University Press: Cambridge, UK, 2013, pp. 77112.

7. Matsuno, K. The internalist stance: a linguistic practice enclosing dynamics. Proc NY Acad Sci 2000, 901, 322-349.

8. Sokolov, Y.N. Higher nervous functions: the orienting reflex. Annu Rev Physiol 1963, 25, 545580.

(C) 2015 by the authors; licensee MDPI and ISIS. This abstract is distributed under the terms and conditions of the Creative Commons Attribution license. 